\title{
Effect of increasing doses of long chain $n-3$ PUFA on vascular function: MARINA - a randomised controlled trial
}

\author{
T. A. B. Sanders ${ }^{1}$, Z. Maniou ${ }^{1}$, W. L. Hall ${ }^{1}$, F. Lewis ${ }^{1}$, P. T. Seed ${ }^{2}$ and P. J. Chowienczyk ${ }^{3}$ \\ ${ }^{1}$ School of Medicine, King's College London, Diabetes and Nutritional Sciences Division, Franklin-Wilkins Building, \\ 150 Stamford Street, London SE1 9NH, UK, ${ }^{2}$ Division of Women's Health, St Thomas' Hospital, London SE1 7EH, UK and \\ ${ }^{3}$ Cardiovascular Division, St Thomas' Hospital, London SE1 7EH, UK
}

Dietary EPA and DHA intakes have been positively associated with improved endothelial function in young adults ${ }^{(1)}$. However, Egert et al. ${ }^{(2)}$ reviewed thirty-three intervention trials investigating the effects of $n-3$ PUFA on fasting and/or postprandial endothelial function and no clear conclusion could be drawn because of the small numbers in many trials and inconsistent methodology. The MARINA study (ISRCTN66664610) was a randomised controlled trial designed to test the hypothesis that increasing doses of EPA and DHA (0.45 g, $0.9 \mathrm{~g}$ and $1.8 \mathrm{~g} / \mathrm{d}$; equivalent to consuming 1,2 or 4 portions of oily fish per week) taken for 1 year have favourable effects on endothelial function measured by flow-mediated dilatation (FMD) of the brachial artery. EPA + DHA and placebo (olive oil BP) were administered as supplements and the study was conducted double blind. A total of 367 non-smoking participants (142 men, 225 women aged 45-70 years, mean age 55 years) were randomised to treatment and a total of 312 subjects completed; data was available for analysis on 311 subjects for baseline and follow-up measurements of endothelial function. The mean $(95 \% \mathrm{CI})$ increase in erythrocyte EPA compared with placebo was $0.74(0.56-0.91), 1.31(1.13-1.48)$ and $2.51(2.33-2.69)$ wt $\%$ on $0.45 \mathrm{~g} / \mathrm{d}, 0.9 \mathrm{~g} / \mathrm{d}$ and $1.8 \mathrm{~g} / \mathrm{d}$, respectively $(P<0.0001)$. Significant reductions in plasma TAG concentrations $(P=0.002)$ were observed in women but not men: in comparison with placebo changes were -0.06 ( -0.21 to 0.09$),-0.12$ ( -0.27 to 0.03$)$ and -0.29 ( -0.44 to -0.14$) \mathrm{mmol} / \mathrm{l}$ on doses of $0.45,0.9$ and $1.8 \mathrm{~g} / \mathrm{d}$, respectively. No significant treatment effects were noted for ambulatory blood pressure or arterial stiffness (pulse wave velocity). FMD was lower in men than in women $(P<0.0001)$ and decreased with age $(\rho=0.270 ; P<0.001)$ but there were no statistically significant treatment effects $(P=0.781)$.

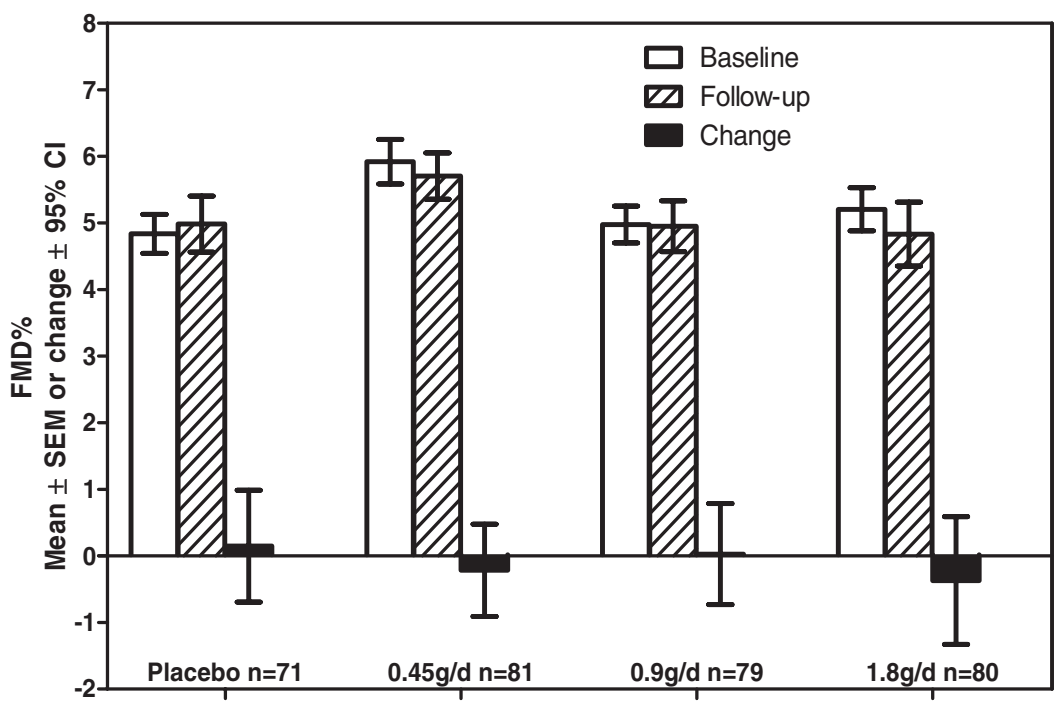

Our results are consistent with a cross-sectional survey in 3045 adults ${ }^{(3)}$ that found no significant relationship between the $n-3$ LCP intake with FMD but a trend for FMD to be 0.1 and $0.27 \%$ lower on the highest compared with lowest intake in men and women, respectively. Our results indicate that intakes of $n-3 \mathrm{LCP}$ up to $1.8 \mathrm{~g} / \mathrm{d}$ do not influence endothelial function in healthy non-smoking adults at moderate risk of CVD.

Funding for this work was provided by the Food Standards Agency and the Department of Health, England (Project code N02041).

1. Leeson CP, Mann A \& Kattenhorn M (2002) Eur Heart J 23, 216-222.

2. Egert S \& Stehle P (2011) Curr Opin Clin Nutr Metab Care 14, 121-31.

3. Anderson JS, Nettleton JA, Herrington DM et al. (2010) Am J Clin Nutr 92, 1204-1213. 